\title{
Advances in research on the pathogenesis of depression based on signaling pathways
}

\author{
Linbo Chen ${ }^{1}$ \\ ${ }^{1}$ College of Life Sciences Sichuan University
}

\begin{abstract}
Depression is an affective disorder that endangers human health, and its main clinical feature is significant and persistent depression. The pathological mechanism of depression is complex and has not been fully elucidated so far. In recent years, with the further study of molecular biology techniques, depression is closely related with mood regulation, cognitive function, function changes of specific protein and abnormal cell signal transduction pathways in the brain, causing cell dysfunction, which affects the brain nerve cells apoptosis regeneration mechanism of trigger depressive symptoms. By referring to and sorting out relevant literatures, this paper comprehensively described the research progress of the signal transduction mechanism of cyclic adenosine phosphate (cAMP), mitogen-activated protein kinase (MAPK) and calmodulin kinase (CaMK) in depression, so as to provide reference for explaining the pathogenesis of depression and developing treatment plans.
\end{abstract}

\section{Introduction}

Depression is a persistent and recurring mood disorder [1]. Its clinical manifestations include low mood, reduced speech and movement, mental retardation, loss of interest in work, self-guilt, self-consciousness of uselessness, and often suicidal tendencies, which are common symptoms that harm all mankind [2]. The signal transduction pathway has the function of cascade amplification, which means one of the most primitive signals can cause the activation of multilevel messengers, which produces the biological effect of tens of thousands of times amplification [3]. Three pathways, namely cyclic adenosine phosphate (cAMP), mitogen-activated protein kinase (MAPK) and calmodulin kinase (CaMK), are currently considered to be related to antidepressant. They constitute the three main upstream pathways of CREB, and ultimately phosphorylate CREB to regulate gene expression in its downstream pathways and complete the biochemical function of neurons [4]. The research progress of signaling pathway in depression is reviewed in this paper, which will provide reference for the explanation of its pathogenesis and the development of treatment plan.

\section{2 cAMP signaling pathway and depression}

Intracellular signal transduction pathway is the focus and difficulty in the current study of antidepressant mechanism. The cAMP Response element binding protein (CREB) is a key protein for many antidepressant drugs and can be activated by many different signaling pathways [5]. CREB plays an extremely important role in the signal transduction pathway. It can connect the upstream and downstream parts of the signal pathway, which are important transportation hubs of the upstream and downstream [6]. Therefore, Cyclic adenosine phosphate (cAMP) is an important second messenger in cells.

\subsection{AC/CAMP/PKA signaling pathway}

AC/cAMP/PKA pathway plays an important role in emotion regulation. Most antidepressants can cause increase of concentrations of 5-hydroxytryptamine (5-HT) and norepinephrine (NE) in the brain. 5-HT and NE bind to g-protein-coupled receptors and activate the cAMP pathway [7]. The main process of the cAMP signaling cascade reaction is as follows: activation of adenylate cyclase (AC) $\rightarrow$ catalysis of ATP to cAMP $\rightarrow$ activation of protein kinase $\mathrm{A}(\mathrm{PKA}) \rightarrow$ phosphorylation of CREB $\rightarrow$ regulation of gene transcription $\rightarrow$ biological effect [8]. Under normal physiological conditions, cAMP is generated by ATP catalyzed by AC and degraded by TYPE IV phosphodiesterase (PDE4) [9]. Jiang H J et al. found that PKA agonist 8-BR-camp could dose-dependently reduce the immobility time of rats in the forced swimming experiment, showing an antidepressant effect. This study proved the antidepressant effect of PKA agonist through animal experiments for the first time[10].Wang etc injected phosphoric acid lipase 2-4 (PDE4) specific inhibitor raleigh alvin (rolipram) which can inhibit cAMP hydrolysis, into mice after continuous dosing 17-23 d finding increase of cAMP, pCREB levels in mice 
hippocampus, reduce of immobile time of the mice in the force swimming test and force tail suspension test in the fixed time, a similar fluoxetine behavior change after the treatment, suggests that the activation of the hippocampus cAMP pathways can improve depressive symptoms, Jiang $\mathrm{H} \mathrm{J}$ also holds this opinion[11]. Kim et al. confirmed that the activity of CAMP and PKA in the prefrontal cortex of suicidal depressed patients was decreased, and the expression of RAP-1, a neuroprotective and synaptic plasticity molecule activated by cAMP or PKA, was also decreased. In addition, in the rat model of acquired helplessness depression, the behavior change of avoiding failure in the rat was related to it[12]. Ringsevgen et al. studied peripheral blood and autopsy brain tissue samples of depressed patients and found that PKA activity was reduced in depressed patients [13]. Therefore, cAMP and its mediators, AC and PKA, which regulate the concentration of cAMP and regulate the role of cells, play a crucial role in the cAMP signaling pathway.

\subsection{5-HT1AR/cAMP/PKA signaling pathway}

It has been reported in the literature that depression is related to the disorder of monoamine neurotransmitter system in brain, especially the central monoamine neurotransmitter[14].5-hydroxy tryptamine 1A receptor may play an important role in the pathogenesis of depression [15].5-HT1AR is a g-protein-coupled receptor, 5-hydroxy Tryptamine is involved in the pathological process of depression through 5-HT1AR mediated C AMP/PKA/CREB signaling pathway.

\subsection{Research on cAMP Pathway and clinical depression}

With the increasing attention paid to the role of cAMP signaling pathway in an animal model, people gradually shifted their attention to the patients with depression-like emotions. In order to explore the effect of SRP on depressed rats, Wei Haojie et al. randomly divided 60 SD rats into 6 groups: normal group, model group, SRP (100, $200,400 \mathrm{mg} / \mathrm{kg})$ group and fluoxetine $(2 \mathrm{mg} / \mathrm{kg})$ group. Different stress methods were used to establish chronic stress depression model in rats, and the changes of behavioral indicators in rats were observed. The content of cAMP was determined by radioimmunoassay, protein kinase A (PKA), and reactive element binding protein (CREB) in the hippocampus were determined by immunohistochemistry[16]. The research results of the experiment shows that the depression model rats after stimulation experiments, depression great heat will appear all sorts of state changes, mainly characterized by typical less activity, also it is because of less activity, reduced rats sugar water consumption and weight loss, integrated the above three kinds of performance, the rats can be judged in a state of depression. Immunohistochemical results showed that, compared with the normal group, the expressions of 5-HT1AR, cAMP, PKA and CREB in the hippocampus of the model group were significantly decreased, while the expressions of 5-HT1AR, cAMP, PKA and CREB in the SRP group were significantly up-regulated $(\mathrm{P}<0.01)$. The data are shown in the figure below:

Effect of SRP on weight of chronic depression rats $(g, n=6, \bar{x} \pm s)$

\begin{tabular}{lccccc}
\hline Group & dosage $(\mathbf{m g} / \mathbf{k g})$ & 1day & 7days & 14days & 21days \\
\hline Regular & - & $167.30 \pm 6.82$ & $205.00 \pm 6.54$ & $231.33 \pm 5.57$ & $290.67 \pm 29.50$ \\
Model & - & $161.90 \pm 6.47$ & $187.50 \pm 5.58^{* *}$ & $182.33 \pm 20.03^{* *}$ & $244.00 \pm 21.16^{* *}$ \\
SRP & 100 & $168.30 \pm 11.23$ & $192.33 \pm 3.93^{* *}$ & $198.00 \pm 11.85^{* *}$ & $276.83 \pm 8.57^{* *}$ \\
& 200 & $167.30 \pm 8.87$ & $194.00 \pm 6.16^{* *}$ & $204.00 \pm 16.80^{* * \#}$ & $296.67 \pm 12.01^{\# \#}$ \\
& 400 & $168.20 \pm 8.55$ & $193.17 \pm 4.79^{* *}$ & $216.83 \pm 16.75^{\# \#}$ & $287.17 \pm 20.93^{* * *}$ \\
Fluoxetine & 2 & $170.40 \pm 12.05$ & $199.83 \pm 7.36^{* \#}$ & $221.33 \pm 12.27^{* \#}$ & $288.00 \pm 14.16^{* *}$ \\
\hline
\end{tabular}

Effect of SRP on sugar water intake in chronic stress rats $(g, n=6, \bar{x} \pm s)$

\begin{tabular}{lccccc}
\hline Group & dosage(mg/kg) & 1day & 7days & 14days & 21days \\
\hline Regular & - & $80.20 \pm 3.55$ & $75.50 \pm 4.97$ & $66.17 \pm 4.02$ & $65.20 \pm 3.39$ \\
Mode1 & - & $81.30 \pm 4.69$ & $72.17 \pm 3.19$ & $52.17 \pm 6.34^{* *}$ & $46.80 \pm 3.19^{* *}$ \\
SRP & 100 & $80.80 \pm 3.68$ & $75.17 \pm 8.16$ & $50.67 \pm 3.67^{* *}$ & $50.10 \pm 4.48^{* *}$ \\
& 200 & $80.40 \pm 3.53$ & $77.83 \pm 7.25$ & $63.17 \pm 5.56^{\text {*\# }}$ & $61.20 \pm 8.34^{\text {*\# }}$ \\
& 400 & $79.80 \pm 3.65$ & $78.80 \pm 4.49$ & $66.50 \pm 4.28^{\text {*\# }}$ & $61.10 \pm 9.64^{\text {*\# }}$ \\
Fluoxetine & 2 & $79.80 \pm 2.90$ & $76.17 \pm 8.57$ & $66.00 \pm 7.29^{\text {*\# }}$ & $62.30 \pm 2.83^{\text {*\# }}$ \\
\hline
\end{tabular}

Effect of SRP on autonomic activity in chronic stress rats $(g, n=6, ; \pm s)$

\begin{tabular}{|c|c|c|c|}
\hline Group & losage $(\mathrm{mg} / \mathrm{kg})$ & 1day & 21days \\
\hline Regular & - & $87.70 \pm 6.29$ & $87.10 \pm 4.65$ \\
\hline Model & - & $86.90 \pm 6.64$ & $61.00 \pm 3.65^{\star *}$ \\
\hline \multirow[t]{3}{*}{ SRP } & 100 & $85.00 \pm 5.39$ & $63.20 \pm 4.31 * *$ \\
\hline & 200 & $85.30 \pm 4.37$ & $82.60 \pm 5.32^{* * n}$ \\
\hline & 400 & $84.90 \pm 6.08$ & $85.30 \pm 5.23^{m W}$ \\
\hline Fluoxetine & 2 & $84.00 \pm 6.58$ & $86.00 \pm 3.29^{\text {类 }}$ \\
\hline
\end{tabular}




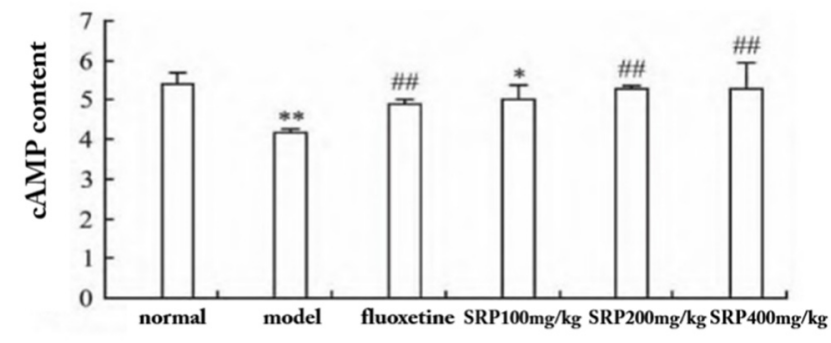

Effect of SRP on cAMP content in hippocampus of chronic stress rats $(n=0, x \pm s)$

Effect of SRP on PKA content in hippocampus of chronic stress rats
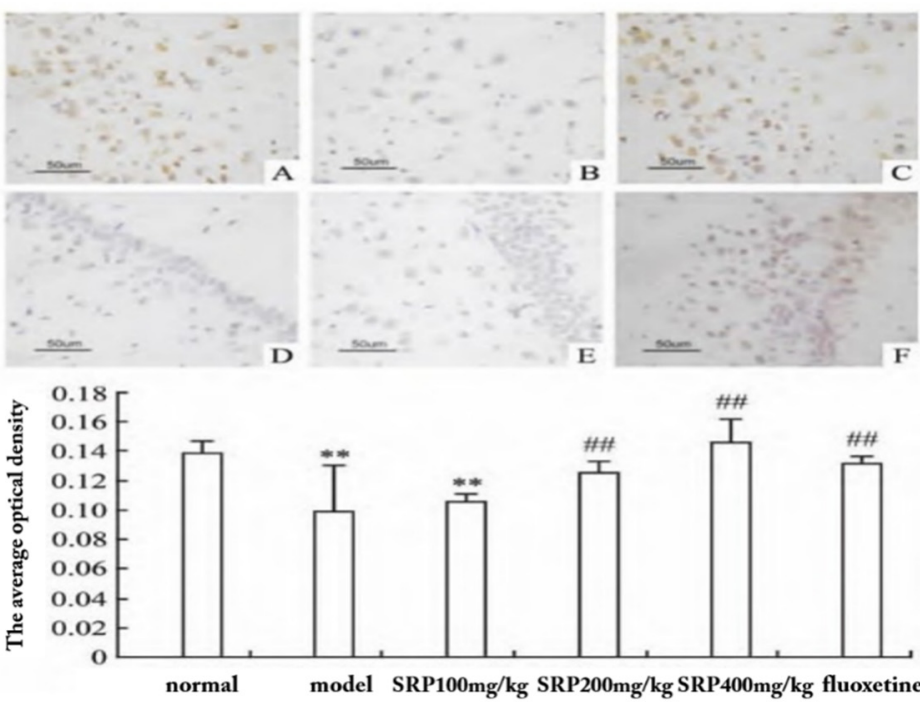

Effect of CREB on content in hippocampus of chronic stress rats

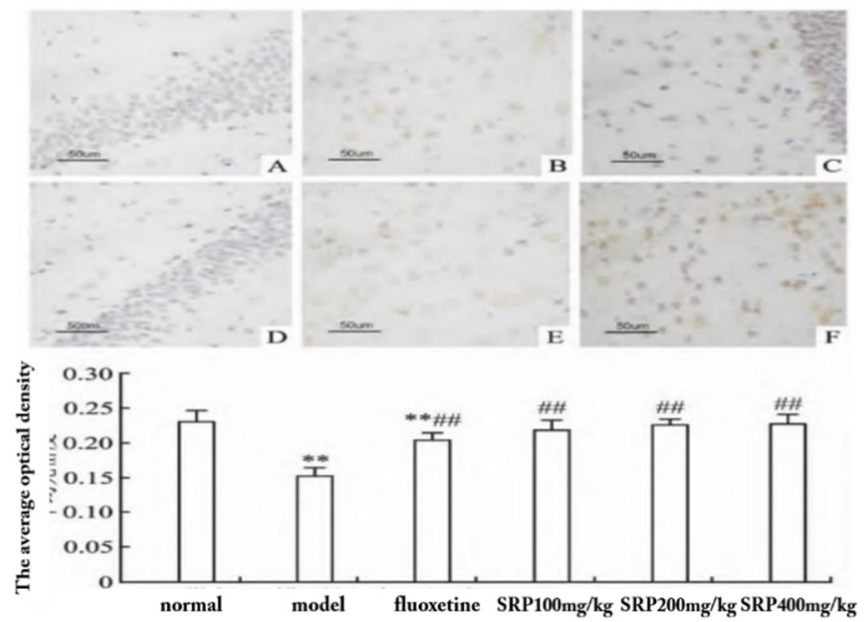

\section{CaMK signaling pathway and depression}

The CaMK pathway plays an important role in the pathogenesis of depression and the antidepressant treatment pathway. Postsynaptic density structure of $\mathrm{Ca} 2$ + / CaMK II signaling pathway is very important. CaMK II is a kind of multi-functional silk/threonine protein kinase, highly expressed in the brain tissue. Lee and others think CaMK II is a high expression of protein kinase, brain tissue, especially the hippocampus area changes in neural plasticity and memory have into with a weight will be used, after CREB is within the nucleus of CaMKII swim points[17]. Phosphorylation of $\mathrm{Ca}^{2+}$-CaM-CaMKII-CREB site is one of a series of cascade reactions in vivo. The activation pathway is as follows: increase of intracellular calcium concentration $\rightarrow$ binding of calcium ions to $\mathrm{CaM} \rightarrow$ activation of 
$\mathrm{CaMK} \rightarrow$ phosphorylation of CREB $\rightarrow$ regulation of gene transcription $\rightarrow$ expression of neurotrophic factors. Studies have found that chronic stress depression can lead to increase of levels of glutamate (GLU) in hippocampal neurons and excessive activation of N-methyl-D-aspartic acid receptor (NMDAR)[18], then the membrane permeability to calcium $\left(\mathrm{Ca}^{2+}\right.$ will increase, causing a large amount of $\mathrm{Ca}^{2+}$ influx leading to intracellular calcium overload, however, Calcium plays an important role in nerve growth and synaptic remodeling[19]. By binding to specific calmodulin ( $\mathrm{CaM})$, calcium ions further activate downstream signaling pathways such as cyclic adenosine reactive element binding protein (CREB) that regulate cognition-related gene transcription, affecting the mood of the living organism. Therefore, changes in $\mathrm{Ca}^{2+}$ concentration are believed to play a key role in depression[20].

\section{MAPK signaling pathway and depression}

The CaMK pathway plays an important role in the pathogenesis of depression and in antidepressant treatment. The $\mathrm{Ca}^{2+} /$ CAMK II signaling pathway is structurally important postsynaptically, and CAMK II manifests itself as a multipurpose serine kinase that is highly frustrated. After MAPK nuclear transposition, it activates intracellular transcription factors, regulates gene expression, promotes the synthesis of related proteins, and completes the response to extracellular stimuli. The basic process of the signaling cascade is as follows: $\mathrm{G}$ protein RAS-activated MAPK kinase (MAP-KKK, RAF) -activated MAP kinase (MAPKK, MEK) -activated extracellular regulatory protein kinase (ERK) 1/2 -activated ribosome S6 kinase (RSK) -CRE phosphorylation - regulation of downstream gene transcription - to produce biological effects. The signal transduction pathway has many functions in neurons, including synaptic plasticity, long-term enhancement effect, protection and survival of damaged neurons [21].

\subsection{ERK1/2 signaling pathway}

Extracellular adjusting protein kinase (ERK1/2) signal transduction pathway is an important subtribe of mitogen-activated protein kinase pathway (MAPKs) family. ERK $1 / 2$ is mainly activated by phosphorylation of various growth factors, ion rays, hydrogen peroxide, etc., enters the nucleus and acts on transcription factors, promotes the transcription and expression of certain genes, and are closely related to cause of the depression[22].

ERK1/2 channel signal cascade follows MAPKs tertiary enzymatic cascade, after the ras/raf/MEK/ERK cascade amplification effect to extracellular signals into the cell, its process is as follows: extracellular signals through the cell membrane surface receptor activation and GTP protein made connection ras proteins, which in turn raf and MEK downstream protein kinase. MEK heterosexual phosphorylation plays an important biological significance, which can effectively prevent ERK1/2 signal error activation, improve the accuracy[23].
The inhibitor PD98059 can specifically inhibit the activation and phosphorylation of MEK1 through binding with ERR, the upstream kinase of ERK1/2 (MEK1 is the main activator of ERK1/2), and thus blocks the ERK1/2 signaling pathway[24]. Part of activated ERK1/2 rapidly enters the nucleus, phosphorylates ribosomal S6 kinase (RSK), and then promotes the phosphorylation of CREB, C-FOS, cJun, ELK-1, NF-XB and other nuclear transcription factors [25]. ERK, which is not activated in the nucleus, stays in the cytoplasm, phosphorylates cytoskeleton proteins, regulates cell morphology and cytoskeletal redistribution, or regulates other protein kinases, mediating other biological effects[26].

ERK1/2 signaling pathway does not exist in isolation. It is cross-linked with other signal transduction pathways and together forms a complex regulatory network to regulate the biological function of cells[27]. For example, the P13K/AKT pathway can affect the cascade effect of the ERK $1 / 2$ pathway, and the interaction between the two signaling pathways is manifested in the form of coordination or withdrawal[28]. The acidification of ERK $1 / 2$ is positively and negatively regulated by a variety of factors[29]. ERK can phosphorylate the upstream proteins of ERK 1/2 pathway, such as Raf-1 and MEK, and further strengthen the MAPK signaling pathway [30], BDNF is the target protein in the downstream of ERK1/2 signaling pathway. Binding with the protein receptor TrkB can activate the ERK signaling pathway and form a circulating pathway[31].

Recent studies have shown that ERK $1 / 2$ activity in the prefrontal cortex and hippocampus is significantly reduced in depressed patients[32].SCHMUNK et al. found that ERK blockers could cause depressed-like behaviors in mice, and could also block the antidepressant effect of antidepressants[33]. KURY et al. found that the ERK signaling pathway in the frontal cortex of rats is correlated to anhedonia and decreased activity level to some extent[34]. COLEY A A et al. found that improving the expression and function of key molecules of BDNF/CREB/ERK signaling pathway in rat brain could effectively improve the mechanism of action of 4ERK $1 / 2$ signaling pathway involved in antidepressant treatment in rat depressive behavior[35].

In conclusion, in nerve cells, activation of ERK1/2 signaling pathway not only has a protective effect, but also has a role of promoting damage, which is a double-edged sword. In order to make good use of the neuroprotective effect of ERK1/2 signaling pathway, specific physiological and pathological regulatory mechanisms of this pathway in different models and different parts must be clarified.

\section{2c-Jun amino-terminal kinase signaling pathway}

JNK and other members of the MAPK family, its substrate molecule phosphorylation of serine/threonine site. JNK family has three subtypes, JNK1, JNK2 and JNK3. They are different from expression in tissue distribution, active regulation and function. JNK1 and 
JNK2 are widely expressed in whole body including the brain, while JNK3 is mainly expressed in the brain.

The involved MAPK kinases include MEKK1, MEKK4, MLK1, MLK3, ASK1, TAOK2, etc., which are known to have important functions in the nervous system[36].A genome-wide expression profile screening study on the hippocampus of mice with unpredictable chronic mild stress depression and mice with isolated mother and child stress depression found that JNk1 (also known as Mapk8) was differentially expressed in both types of depressed mice compared with the control group[37].

\subsection{MAKP pathway and clinical depression}

With the increasing attention paid to the role of MAKP signaling pathway in an animal model, people gradually shifted their attention to the patients with depression-like emotions. Xu Ming-min ( 2016 ) [38]specifically inhibited the ERK1/2 signaling pathway and to observe the changes in protein expression levels of key effectors p-ERK1/2, P-CREB and BDNF in the erK $1 / 2$ creB-BDNF signaling pathway in the prefrontal cortex of rats with chronic stress depression. Experimental methods: 64 SD rats dimensionality, randomly divided into 8 groups, each group of eight: blank group, model group, model plus acupuncture group (acupuncture group), model + chlorine west group (fluoxetine group), model group, the model ++ DMSO PD98059 + + PD group (model), model group acupuncture group (acupuncture + PD) + PD98059 group + + PD98059 fluoxetine group,

\begin{tabular}{cccc}
\multicolumn{4}{c}{$\begin{array}{c}\text { After the experiment, the protein expression of ERK1/2 and P- } \\
\text { ERk } 1 / 2 \text { in the prefrontal cortex of each group was compared }\end{array}$} \\
\hline group & $\mathrm{n}$ & ERK1 $1 / 2$ & P-ERK $1 / 2$ \\
\hline normal & 6 & $0.581 \pm 0.143$ & $0.428 \pm 0.079$ \\
model & 6 & $0.558 \pm 0.151$ & $0.207 \pm 0.049^{\star \star}$ \\
fluoxetine & 6 & $0.585 \pm 0.134$ & $0.347 \pm 0.071^{\star \star}$ \\
Acupuncture & 6 & $0.595 \pm 0.136$ & $0.309 \pm 0.072^{\star \star}$ \\
model +DMSO & 6 & $0.574 \pm 0.146$ & $0.248 \pm 0.039^{\star \star}$ \\
model +PD & 6 & $0.594 \pm 0.138$ & $0.226 \pm 0.079^{\star \star}$ \\
Fluoxetine+PD & 6 & $0.594 \pm 0.139$ & $0.252 \pm 0.056^{\circ}$ \\
Acupuncture+PD & 6 & $0.580 \pm 0.160$ & $0.199 \pm 0.043^{* *}$ \\
\hline
\end{tabular}

Note: Compared with the blank group, ${ }^{\mathrm{P}}<\mathbf{0 . 0 1}$. Compared with the model group, $\triangle \mathbf{P}<0.01$, compared with the acupuncture group, $\bullet \mathbf{P}<0.01$, compared with the fluoxetine group, $\diamond \mathbf{P}<\mathbf{0 . 0 5}$.

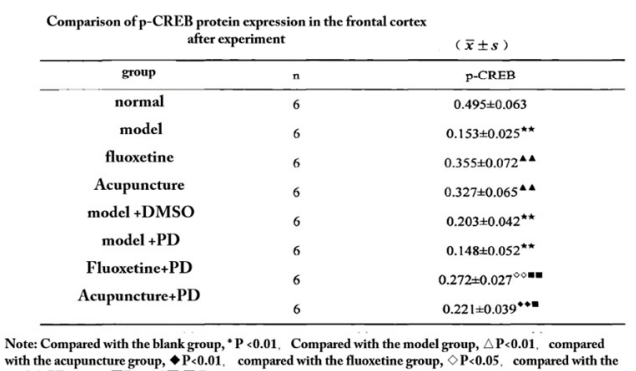

model group chlorine paxil + (PD). Modeling methods: The depression rat model was prepared by the combination of solitary nutrition and chronic mild unforeseeable stress. Stress management involves a 24-hour diet. Cut off the water for $24 \mathrm{~h}$; Binding $2 \mathrm{~h}$; vibration for 30 minutes; Editing for 3 minutes; The wet mat for 24h: $12 \mathrm{~h}$ of night-light exposure, the stress stimulation averaged 3 times during the experiment, the same stress pattern was used intermittently, a total of 21 days of modeling. 1 week before model preparation, lateral ventricular vesicle management was performed in model +DMSO group, model + PD group, acupuncture + PD group and fluoxetine +PD group. Normal group did not give any stimulation, normal group feeding. The other groups received chronic stress stimulation for 21 days and were fed in solitary cages. At the same time, the acupuncture group received acupuncture treatment $30 \mathrm{~min}$ before modeling every day: the fluoxetine group received gastric administration $30 \mathrm{~min}$ before stress every day: the model +DMSO group was given 10UL/LD DMSO lateral ventricle injection $1 \mathrm{~h}$ before stress: the model +PD group was given 10UL / 1D blocking inhibitor PD98059 diluent lateral ventricle injection $1 \mathrm{~h}$ before stress: The acupuncture $+\mathrm{PD}$ group and the cloxetine +PD group were injected with 10UL/LD blocking inhibitor PD98059 diluent in the lateral ventricle $1 \mathrm{~h}$ before the stress, and then acupuncture intervention and fluoxetine gavage administration were respectively administered 30min before the stress. Experimental results are shown in the following table:

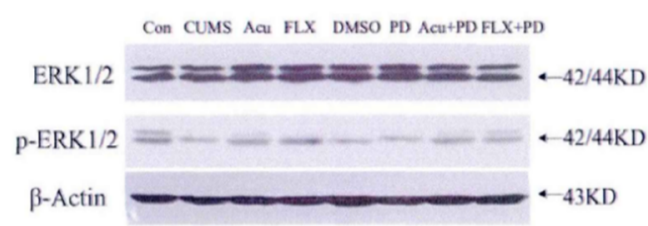

The expression of ERK1/2 and P-ERk1/2 in the prefrontal cortex of rats in each group

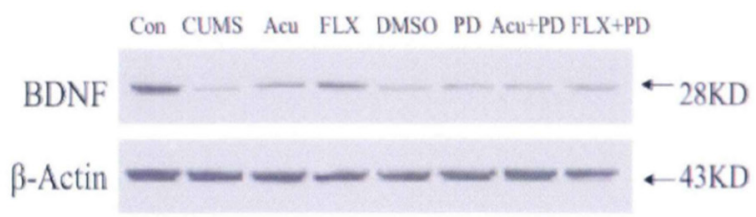

BDNF protein expression in frontal lobe of each group 


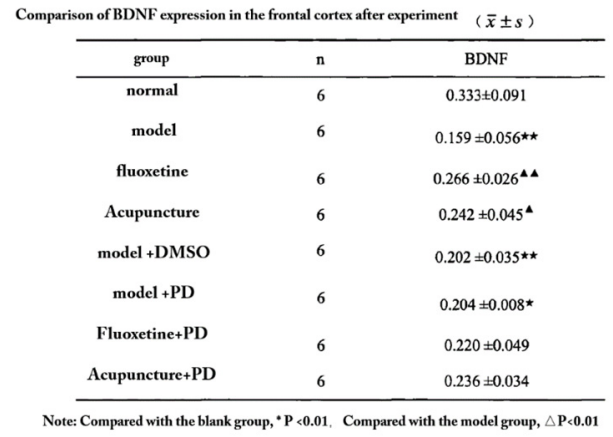

From the above experimental data, it can be found that there is no significant difference in the expression of ERK1/2 protein in the prefrontal cortex between each group of experimental rats. C U M S combined with any other ingredients, can make the related research in the prefrontal cortex of the factor content is reduced, the protein expression decrease, means that use this way to be able to make the expression of these proteins by will, it is possible that inhibit the synthesis of these proteins, or blocking the protein synthesis pathway, according to the related research content of this study may prove that chronic stress stimulation inhibits the activity of ERK1/2 signaling pathways. Through longitudinal comparison of acupuncture group and acupuncture + PD composite, can be found, as the hub of the signal factor BDNF not appear relatively obvious change, while the other two protein expression of all appear to drop the case, fluoxetine group with fluoxetine + PD group have the same performance, the two groups can be fully proved blocker PD98059 inhibition, but these two approaches for transport hub of BDNF protein, no intervention. In addition, acupuncture and fluoxetine interventions have been shown to up-regulate CREB phosphorylation through other pathways, while oxoxetine may be more regulated through other pathways.

\section{5 conclusion}

The pathogenesis of depression is very complex, and the causes of depression are also various, including many factors such as environment, living habits, heredity and dietary habits. Research evidence tends to be related to the dysfunction of various neurotransmitters and receptors, neuroendocrine and BDNF abnormalities in the body. At the same time, the occurrence of depression may be related to the impairment of neuroperception function and the change of neuroelectrophysiology. Its abnormality involves multiple signaling pathways of life activity, and the pathogenesis of depression cannot be well elucidated only from one aspect, but its correlation has not been elucidated at present. Therefore, synthesizing various hypotheses and phenomena and finding out the key points of intersection is an important method to elucidate depression.

\section{reference}

1. Alberich S, Martínez-Cengotitabengoa M, López P.Efficacy and safety of ketamine in bipolar

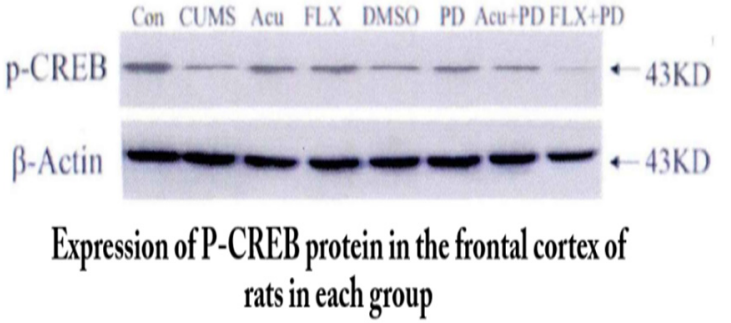

depression:A systematic review[J].Rev Psiquiatr Salud Ment, 2017, 10 (2) :104-112.

2. Ren $\mathrm{W}$, Luan $\mathrm{X}$, Zhang $\mathrm{J}$, et al.Brain-derived neurotrophic factor levels and depression during methamphetaminewithdrawal[J].J Disorders, 2017, 15 (221) :165-171.

3. Zannas AS, Wiechmann T, Gassen NC, et al.Genestress-epigenetic regulation of FKBP5:clinical and translational implications[J].Neuropsychopharmacology, 2016, 41 (1) :261-274.

4. Yang Y, Cui Y, Sang K, et al.Ketamine blocks bursting in the lateral habenula to rapidly relieve depression[J].Nature, 2018, 554 (7692) :317-322.

5. Mrazek DA, Hornberger JC, Altar CA, et al.A Review of the clinical, economic, and societal burden of treatment-resistant depression: 1996-2013 [J].Psychiatric Services, 2014, 65 (8) :977-987.

6. 10 Song M, Martinowich K, Lee FS. BDNF at the synapse: why location matters[J].Molecular Psychiatry, 2017, 22 (10):1370-1375.

7. ROUBERTOUX P L, TORDJMAN S, CAUBIT X, et al.Construct validity and cross validity of a test battery modeling autism spectrum disorder (ASD) in mice[J].Behav

Genet,2020,50(1):26-40.DOI:10.1007/s10519-019-0 9970-X.

8. DE RUBEIS S, HE X, GOLDBERG A $\mathrm{P}$, et al.Synaptic, transcriptional and chromatin genes disrupted autism[J].Nature,2014,515(7526):209-215.DOI:10.1 038/nature13772.

9. YANG Y R, KANG D S, LEE C, et al.Primary phospholipase $\mathrm{C}$ and brain disorders[J].Adv Biol Regul,2016,61:80-85.DOI:10.1016/j.jbior.2015.11.0 03.

10. JANG H J, SUH P G, LEE Y J,et al.PLC $\gamma 1$ :Potential arbitrator of cancer progression[J].Adv Biol Regul,2018,67:179-189.DOI:10.1016/j.jbior.2017.11 .003 .

11. WANG $\mathrm{M}$ J, JIANG L, CHEN $\mathrm{H}$ S, et al.Levetiracetam protects against cognitive impairment of subthreshold convulsant discharge model rats by activating protein kinase $\mathrm{C}$ (PKC)-growth-associated protein 43 (GAP-43)-calmodulin-dependent protein kinase $(\mathrm{CaMK})$ signal transduction pathway[J]. Med Sci 
Monit, 2019, 25: 4627-4638.DOI: 10.12659/MSM.913542.

12. KIM H Y, YANG Y R, HWANG H, et al.Deletion of PLC $\gamma 1$ in GABAergic neurons increases seizure susceptibility in aged mice[J].Sci Rep, 2019, 9(1): 17761.DOI: 10.1038/s41598-019-54477-4.

13. RINGSEVJEN $\mathrm{H}$, UMBACH HANSEN H M, HUSSAIN S, et al.Presynaptic increase in IP3 receptor type 1 concentration in the early phase of hippocampal synaptic plasticity[J].Brain Res,2019,1706:125-134.DOI:10.1016/j.brainres.201 8.10 .030 .

14. Landolt H P, Wehrle R.Antagonism of serotonergic 5-HT2A/2Creceptors:mutual improvement of sleep, cognition and mood?[J].Eur J Neurosci, 2009, 29 (9) $: 1795$.

15. Alexandre C, Popa D, Fabre V, et al.Early life blockade of 5-hydroxytryptamine 1A receptors normalizes sleep and depression-like behavior in adult knock-out mice lacking the serotonin trans-porter[J].J Neurosci, 2006, 26 (20) :54-5.

16. Wei Haojie; Wei-ping xu; Wei; Chen Cheng. Effect of Huangjing saponins on 5-HT1AR/cAMP/PKA signaling pathway in hippocampus of rats with chronic stress depression. Journal of Anhui Medical University.2012 (05):38-42

17. Lee SJ, Kim HE, Choi SE, et al. Involvement of $\mathrm{Ca}$ in genistein-induced potentiation of leucine/glutamine-stimulated insulin secretion[J].Molcells,200928(3):167-174

18. Benito E, Barco A.CREB's control of intrinsic and synaptic plasticity:implications for CREB-dependent memory models[J].Trends Neurosci, 2010;33 (5) :230-40.

19. Fu H, Li W, Lao Y, et al.Bis (7) -tacrine attenuates beta amyloid-induced neuronal apoptosis by regulating L-type calcium channels[J].JNeurochem, 2006;98 (5) :1400-10.

20. Cross JL, Meloni BP, Bakker AJ, et al.Modes of neuronal calcium entry and homeostasis following cerebral ischemia[J].Stroke Res Treat, 2010;2010:316862.

21. Li H, Zhang L, Huang Q.Differential expression of mitogen-activatedprotein kinase signaling pathway in the hippocampus of rats exposed tochronic unpredictable stress[J].Behav Brain Res, 2009, 205 (1) :32-37.

22. TANG G M, GUDSNUK K, KUO S H, et al.Loss of mTOR-dependent macroautophagy causes autistic-like synaptic pruning deficits[J].Neuron,2014,83(5):1131-1143.DOI:10.10 16/j.neuron.2014.07.040.

23. MARKRAM K, RINALDI T, LA MENDOLA D, et al.Abnormal fear conditioning and amygdala processing in an animal model of autism[J].Neuropsychopharmacology,2008,33(4):90 1-912.DOI:10.1038/sj.npp.1301453.

24. HARA Y, AGO Y, TARUTA A, et al.Risperidone and aripiprazole alleviate prenatal valproic acid-induced abnormalities in behaviors and dendritic spine density in mice[J].Psychopharmacology (Berl),2017,234(21):3217-3228.DOI:10.1007/s0021 3-017-4703-9.

25. YAMAGUCHI H, HARA Y, AGO Y, et al.Environmental enrichment attenuates behavioral abnormalities in valproic acid-exposed autism model mice[J].Behav Brain Res,2017,333:67-73.DOI:10.1016/j.bbr.2017.06.035

26. YANG Y R, JUNG J H, KIM S J,et al.Forebrain-specific ablation of phospholipase $C \gamma 1$ causes manic-like behavior[J].Mol Psychiatry,2017,22(10):1473-1482.DOI:10.1038/mp .2016.261.

27. SHIMOHAMA S, SUMIDA Y, FUJIMOTO S, et al.Differential expression of rat brain phospholipase $\mathrm{C}$ isozymes in development and aging $[\mathrm{J}]$.Biochem Biophys Res Commun,1998,243(1):210-216.DOI:10.1006/bbrc.1 998.8090 .

28. GIRALT A, RODRIGO T, MARTÍN E D, et al.Brain-derived neurotrophic factor modulates the severity of cognitive alterations induced by mutant huntingtin: involvement of phospholipase $\mathrm{C}$ gamma activity and glutamate receptor expression[J].Neuroscience, 2009, 158(4): 1234-1250.

DOI: 10.1016/j.neuroscience.2008.11.024.

29. SHARP A H, NUCIFORA F C Jr, BLONDEL O, et al.Differential cellular expression of isoforms of inositol 1,4,5-triphosphate receptors in neurons and Glia in brain[J].J Comp Neurol, 1999, 406(2):207-220.

30. TAKEMOTO-KIMURA S, SUZUKI K, HORIGANE S I, et al.Calmodulin kinases: essential regulators in health and disease[J].J Neurochem, 2017, 141(6): 808-818.DOI: 10.1111/jnc.14020.

31. XU J, LIU Z A, PEI D $\mathrm{S}$, et al.Calcium/calmodulin-dependent kinase II facilitated GluR6 subunit serine phosphorylation through GluR6-PSD95-CaMKII signaling module assembly in cerebral ischemia injury[J].Brain Res, 2010, 1366: 197-203.DOI: 10.1016/j.brainres.2010.09.087.

32. PENZES P, CAHILL $M$ E, JONES $K A$, et al.Convergent CaMK and RacGEF signals control dendritic structure and function[J].Trends Cell Biol, 2008, 18(9): 405-413.DOI: 10.1016/j.tcb.2008.07.002.

33. SCHMUNK G, BOUBION B J, SMITH I F, et al.Shared functional defect in IP3 R-mediated calcium signaling in diverse monogenic autism syndromes[J].Transl Psychiatry, 2015, 5: e643.DOI:10.1038/tp.2015.123.

34. KÜRY S, VAN WOERDEN G M, BESNARD T, et al.De novo mutations in protein kinase genes 
CAMK2A and CAMK2B cause intellectual disability $[\mathrm{J}] . A m \quad \mathrm{~J}$ Hum Genet,2017,101(5):768-788.DOI:10.1016/j.ajhg.201 7.10.003.

35. COLEY A A, GAO W J.PSD95: a synaptic protein implicated in schizophrenia or autism?[J].Prog Neuropsychopharmacol Biol Psychiatry,2018,82:187-194.DOI:10.1016/j.pnpbp.2 017.11.016.

36. Rodrigues R, Petersen R B, Perry G.Parallels between major depressive disorder and Alzheimer's disease: Role of oxidative stress and genetic vulnerability. Cell Mol Neurobiol, 2014, 34:925-949

37. Malki K, Keers R, Tosto G M, et al. The endogenous and reactive depression subtypes revisited: Integrative animal and human studies implicate multiple distinct molecular mechanism underlying major depressive disorder. BMC Med, 2014, 12:73-86

38. $\mathrm{Xu}$ Ming-min. Effect of acupuncture on erK1/2 -CREB-BDNF signaling pathway in prefrontal cortex of depressed rats. Beijing University of Chinese Medicine.2016 (08) 